\title{
Editorial: Fractional Calculus and Its Applications in Physics
}

\author{
Devendra Kumar ${ }^{1 *}$ and Dumitru Baleanu ${ }^{2,3}$ \\ ${ }^{1}$ Department of Mathematics, University of Rajasthan, Jaipur, India, ${ }^{2}$ Department of Mathematics, Faculty of Arts and \\ Sciences, Cankaya University, Etimesgut, Turkey, ${ }^{3}$ Institute of Space Sciences, Măgurele, Romania
}

Keywords: fractional calculus, mathematical models, fractional order differential equations, Newell-WhiteheadSegel equation, Laguerre differential equation, Hilfer-Prabhakar fractional operator

\section{Editorial on the Research Topic}

\section{Fractional Calculus and Its Applications in Physics}

Fractional calculus is deeply related to the dynamics of complicated real-world problems. Fractional operators are non-local and describe several natural phenomena in a better and systematic manner. Many mathematical models are accurately governed by fractional order differential equations. Since the classical mathematical models are special cases of the fractional order mathematical models, it implies that the results for the fractional mathematical model are more general and more accurate. The fractional derivatives and integrals are very helpful for engineers, mathematicians, scientists, and researchers working with the real-life phenomena. Thus, this Research Topic Ebook contains some recent investigations demonstrating the depth and breadth of ongoing studies in the area of fractional calculus and its applications in physics. It contains nine articles from 40 authors from all over the world.

Prakash and Verma presents a user-friendly technique using the theory of Adomian decomposition technique to obtain the analytical solutions of the Newell-Whitehead-Segel equations of fractional order. The fractional Newell-Whitehead-Segel equation finds its applications to interpret the formation of the stripe patterns in two-dimensional (2-D) systems. The authors show that the numerical results derived with the aid of the suggested scheme are very accurate.

Shat et al. present a fractional extension of the Laguerre differential equation. The authors used the conformable derivative of order $0<\alpha<1$. The authors used the Frobenius scheme together with the fractional power series expansion to derive two linearly independent solutions of the problem. The authors derive the fractional Laguerre functions in closed forms, and establish their orthogonality results.

Gill et al. show the computable solution of the advection-dispersion equation of the arbitrary order pertaining to Hilfer-Prabhakar fractional operator as well as the Laplace operator of fractional order. The technique for obtaining the solution is a mixed approach using the application of Sumudu and Fourier transforms. The authors derive the solution in compact and graceful forms in the form of the generalized Mittag-Leffler function, which is compatible for numerical evaluation of the results.

Hristov presents both the theory and formulation of linear viscoelastic response functions and their reasonable connection with the Caputo-Fabrizio (CF) fractional operator by using the Prony series decomposition (PSD). The author discusses the problem of interconversion with power and exponential laws and pays very special attention on the PSD approach, the connected interconversion problems, and the presentation of the viscoelastic constitutive equations in the form of CF operator of arbitrary order.

Cattani extends the sinc-fractional derivative to the Hilbert space established on Shannon wavelets. The author defines some novel operators of arbitrary order by using the concept of 
wavelets. The author's main work is to study the localization and compression nature of wavelets when working with operators of non-integer order.

Turalska and West present mathematical concept of the dynamical decision-making model and renewal events, and subordinate the nature of the individual to the mean field nature of the network. The authors proved that the dynamics of the individual is obtained by using the theory of subordination to be a tempered fractional differential equation. The authors reported the exact solution of fractional differential equation in the form of Mittag-Leffler function and computed the numerical results.

Chen et al. show that the Lévy flight is more effective than the Brownian motion if the targets are sparse. The authors consider that every flight of the forager is possibly interrupted by some unknown factors, such as hurdles on the direction of flight, natural opponents in the vision distance, and limitations in the energy storage for every flight, and suggested the tempered Lévy distribution $p(l) \sim \mathrm{e}^{-\rho l} l^{-\mu}$. The authors validate both theoretical inspection and simulation outcomes and that a higher searching coherence can be achieved if the lower values of $\rho$ or $\mu$ are selected. The authors demonstrate that by considering the flight time as the waiting time, the master equation (ME) of the random searching procedure can be determined. The authors construct two distinct kinds of MEs: one is the classical diffusion equation and second is the tempered diffusion equation of fractional order.

Agarwal et al. apply the fractional operators suggested by Marichev-Saigo-Maeda containing Appell function and establish many novel results of extended Lommel-Wright function. The authors also report that by using some integral transforms on the derived results, many more new and known results can be obtained.

Calcagni discusses the recent plans and agendas to be carried out in order to establish a workable definition of scale-dependent fractional operators and their applications to field theory and gravity. The author investigates distinct kinds of multifractional Laplacians and their properties.

\section{AUTHOR CONTRIBUTIONS}

All authors listed have made a substantial, direct and intellectual contribution to the work, and approved it for publication.

\section{ACKNOWLEDGMENTS}

We are highly thankful to all the contributors, reviewers, and editors that have contributed to this Research Topic.

Conflict of Interest Statement: The authors declare that the research was conducted in the absence of any commercial or financial relationships that could be construed as a potential conflict of interest.

Copyright (C) 2019 Kumar and Baleanu. This is an open-access article distributed under the terms of the Creative Commons Attribution License (CC BY). The use, distribution or reproduction in other forums is permitted, provided the original author(s) and the copyright owner(s) are credited and that the original publication in this journal is cited, in accordance with accepted academic practice. No use, distribution or reproduction is permitted which does not comply with these terms. 